\title{
La enfermedad renal crónica en Colombia: necesidades en salud y respuesta del Sistema General de Seguridad Social en Salud*
}

\section{Chronic Kidney Disease in Colombia: Health Needs and Response of the General System of Social Security in Health}

\section{Doença Renal Crônica na Colômbia: necessidades em saúde e de resposta do Sistema Geral de Segurança Social em Saúde}

Fecha de recepción: 15-01-16 Fecha de aceptación: 20-04-16 Disponible en línea: 03-05-16 doi:10.11144/Javeriana.rgyps15-30.ercc

Cómo citar este artículo:

Lopera-Medina MM. La enfermedad renal crónica en Colombia: necesidades en salud y respuesta del Sistema General de Seguridad Social en Salud. Rev. Gerenc. Polit. Salud. 2016; 15(30): 212-233. http:// dx.doi.org/10.11144/Javeriana.rgyps15-30.ercc

Mónica María Lopera-Medina **

Ensayo. Esta investigación es uno de los productos derivados de la investigación "Caracterización clínica, sociodemográfica y financiera de los eventos de alto costo en Antioquia (2012-2013)", financiada por el Fondo de Apoyo Docente de la Facultad Nacional de Pública, Universidad de Antioquia y la Estrategia de Sostenibilidad del Comité para el Desarrollo de la Investigación (CODI) de la Universidad de Antioquia 2012-2013. Reino Unido. Autor principal y responsable de la comunicación. Dirección: Calle 62 N. ${ }^{\circ}$ 52-59 Bloque 33 Oficina. Correo electrónico: monica.lopera@udea.edu.co. Conflicto de interés: ninguno. 


\section{Resumen}

La enfermedad renal crónica (ERC) es una enfermedad de alto costo, progresiva, no trasmisible y estrechamente ligada a otras enfermedades, como las cardiovasculares (ECV) y la diabetes mellitus (DM). La incidencia, prevalencia y mortalidad de la ERC va en aumento en Colombia, a pesar de que existen políticas que orientan el proceso de prevención y manejo. En el Sistema General de Seguridad Social en Salud, la ERC se ha manejado bajo un modelo de atención individual y biomédico que desestima la importancia de la prevención de la enfermedad. La autoridad sanitaria no ejerce eficazmente la vigilancia y control de los actores y privilegia la compensación económica a las Empresas Promotoras de Salud (EPS), sin que medie una verdadera evaluación de los resultados en salud. Este ensayo muestra la situación de la ERC en el país y la respuesta del SGSss, con el propósito de incentivar la reflexión hacia la necesidad de introducir un nuevo modelo de atención.

Palabras clave: insuficiencia renal crónica; gastos en salud; prevención y control; sGSSS

\section{Abstract}

Chronic Kidney Disease (CKD) is a high-cost illness, non-transmissible and progressive disease, closely related to others diseases such as cardiovascular ones and diabetes. Incidence, prevalence and mortality are increasing in Colombia, despite the existence of policies tempting to orientate the prevention, and healthcare process. In The General Social Security System, CKD has been treated preferably under a model of individual and biomedical attention that dismisses the importance of prevention of the disease. The health authority seems to be weak to exert an actual surveillance and control of actors involved in the System, and encourage economic compensation to Health Insurance Companies without taking into account an actual assessments of health results. This essay shows both, CKD situation in the country, and the response of the General System of Social Security in Health, with the purpose of encourage the reflection on the need to introduce a new model of healthcare.

Keywords: Renal Insufficiency; Chronic; Health Expenditures; Cost Allocation; Social Security System in Colombia

\section{Resumo}

A Doença renal crônica (DRC) é uma doença de alto custo, progressiva, não transmissível e intimamente ligada a outras patologias, tais como as cardiovasculares (DCV) e a diabetes mellitus (DM). A incidência, prevalência e mortalidade da DRC estão aumentando na Colômbia, embora existam políticas que orientem o processo de prevenção e gestão. No Sistema Geral de Segurança Social em Saúde (SGSSS), a DRC tem sido gerida sob um modelo de atenção individual e biomédico descartando a importância da prevenção de doenças. A autoridade de saúde não exerce efetivamente a fiscalização e o controle dos atores, e ainda privilegia a compensação econômica para as companhias de seguros da Saúde (EPS), sem que haja uma verdadeira avaliação dos resultados em saúde. Nesta dissertação pretende-se mostrar a situação da DRC na Colômbia, e a resposta do sGSSS, com o objetivo de incentivar a reflexão sobre a necessidade.

Palavras-chave: Insuficiência Renal crônico; Gastos em Saúde; prevenção \& controle; sGsss 


\section{Introducción}

La enfermedad renal crónica (ERC) es una enfermedad progresiva, no trasmisible y estrechamente ligada a otras enfermedades, como las cardiovasculares (ECV) y la diabetes mellitus (DM). A pesar de su carácter prevenible, su incidencia está en aumento en todo el mundo, principalmente en épocas de globalización, en las que se han introducido nuevos factores de riesgo derivados de la producción y consumo, riesgos que se han sumado a la pobreza e inequidad social y sanitaria.

La complejidad de su manejo deriva de varios factores interrelacionados: 1) su complejidad clínica y psicosocial, que demanda largos, complejos y costosos tratamientos para un creciente número de afectados, y por lo tanto es una enfermedad de alto costo para el sistema de salud; 2) en el sistema de salud colombiano, la enfermedad se ha manejado preferentemente dentro de un modelo de atención individual y biomédico que desestima la importancia de la promoción de la salud y la prevención de la enfermedad; 3) en relación con lo anterior, la política incentiva la compensación económica a las EPS en razón de la atención del alto costo, en lugar de incentivar los resultados alcanzados en términos de salud y procesos preventivos; 4) aún si se analiza desde el paradigma biomédico, la organización del sistema de salud presenta importantes fallas para lograr el diagnóstico y la atención oportuna, lo que aumenta el riesgo de progresión y genera mayor carga de enfermedad, al tiempo que disminuye la calidad de vida de los pacientes; y 5) el debilitamiento de la función rectora de la autoridad sanitaria, que delega la vigilancia en salud pública y la gestión financiera de las enfermedades catastróficas en terceros, lo que permite la escalada de costos de medicamentos e insumos. Este 214 ensayo pretende mostrar la situación de la ERC en el país, y la respuesta que el sGsss ha dado a esta compleja enfermedad, y con base en ello propone la reflexión acerca del papel de los actores sociales y de la necesidad de cambiar el modelo sanitario.

\section{Características biomédicas y psicosociales de la ERC}

La enfermedad renal crónica (ERC) es una de las enfermedades no trasmisibles que, a pesar de ser prevenibles, están ocasionando mayor mortalidad en mundo. En esta se incluye un grupo de enfermedades heterogéneas cuyas manifestaciones y curso clínico dependen de la causa y el tipo de afectación, la gravedad, la tasa de progresión y las comorbilidades (1). La ERC se define como una disminución lenta, progresiva e irreversible en la función renal, asociada directamente a la acumulación de complicaciones (2). Se considera como el daño renal o TFG inferior a $60 \mathrm{ML} / \mathrm{min} / 1,73$ $\mathrm{m}^{2}$ durante más de tres meses. Se clasifica en cinco estadios progresivos (ERC-1-ERC-5), según las guías K/DOQI publicadas en el 2002 por la National Kidney Foundation (3) y refrendadas en el 2015 (4). Estos estadios se basan en el grado de disminución de la función renal, valorada por la alteración en la tasa de filtrado glomerular (TFG). La TFG en el estadio 1 (ERC-1) puede ser normal o alta $\left(\geq 90 \mathrm{ML} / \mathrm{min} / 1,73 \mathrm{~m}^{2}\right)$; en el estadio 2 (ERC-2) puede oscilar entre 60 y $80 \mathrm{ML} /$ $\mathrm{min} / 1,73 \mathrm{~m}^{2}$; en el estadio 3 (ERC-3) entre 30 y $59 \mathrm{ML} / \mathrm{min} / 1,73 \mathrm{~m}^{2}$; en el estadio 4 (ERC-4) entre 15 y $29 \mathrm{ML} / \mathrm{min} / 1,73 \mathrm{~m}^{2}$, y en el estadio 5 (ERC-5) se produce falla renal y la TFG es inferior a $15 \mathrm{ML} / \mathrm{min} / 1,73 \mathrm{~m}^{2}(5)$.

Debido a la falla renal severa que acontece en la ERC-5, clínicamente se requieren tratamientos complejos con terapias de sustitución (diálisis o trasplante). Este estadio ha sido mal llamado enfermedad renal 
crónica terminal (ERCT). ${ }^{1}$ En esta condición, la enfermedad no solo afecta los procesos psicofísicos del individuo, sino que también impone grandes y crecientes costos, tanto para el sistema de salud como para el paciente y su familia $(6,7)$. Desde un punto de vista sociosanitario, es una de las enfermedades que provocan más años de vida perdidos (8).

Por su complejidad y progresividad, la ERC obliga al paciente a someterse a constantes tratamientos clínicos y analíticos, a llevar una dieta limitada y a consumir permanentemente medicamentos (9). Además de verse afectadas las funciones física y cognitiva (10), la ERC genera una disminución en la calidad de vida del paciente, de su familia y del entorno laboral, al afectar sus funciones social, económica y emocional (11). Puede desencadenar fenómenos emocionales como estrés, angustia, miedo, ansiedad y depresión. Esta complejidad obliga a que el enfoque de atención —más allá del tratamiento médico oportuno y completo-incorpore elementos que permitan mejorar el bienestar percibido por el paciente (9). La ERC amenaza la esperanza de vida de las personas que la padecen, por la probabilidad de enfermedad propia de la disfunción renal y la aparición de otras patologías asociadas a ella.

La ERC se considera un problema de salud pública y una enfermedad emergente (8), debido a que su frecuencia ha sido creciente en las últimas décadas y a que, con ello, se han producido importantes consecuencias clínicas, sociodemográficas, epidemiológicas y económicas para los individuos y los siste-

1 Aunque esta denominación persiste en el argot médico, el concepto de enfermedad terminal ha sido recientemente cuestionado, por la carga emocional y la estigmatización que produce en el paciente que la padece y en su grupo familiar. Adicionalmente, se ha descrito que esta determinación podría influir negativamente en el tipo de cuidados que se proveen. En su lugar, se propone hablar de paciente con enfermedad avanzada (PEA). mas de salud. En el sistema colombiano se ha reconocido como una enfermedad de alto costo (EAC), catastrófica o ruinosa, porque es de larga duración e implica un manejo de alta complejidad técnica, en especial en los estadios más avanzados (12).

\section{Epidemiología de la ERC en Colombia: factores de riesgo y enfermedades relacionadas}

La prevalencia e incidencia de la ERC en Colombia no se conoce con exactitud, pero se calcula que ha tenido un aumento progresivo, debido a su asociación con múltiples factores de riesgo. En el país, las principales causas de ERC las constituyen las enfermedades precursoras como la hipertensión arterial (HTA) y otros tipos de enfermedad cardiovascular (ECV), la diabetes mellitus tipo II y la uremia (5). Otras enfermedades asociadas con la ERC son las enfermedades autoinmunes, la insuficiencia renal aguda y el VIH. En estos pacientes se recomienda efectuar estudios de cribaje para detección oportuna de la ERC $(13,14)$. Las principales causas de insuficiencia renal crónica (IRC) en niños están relacionadas con reflujo vésicoureteral, hidronefrosis, valvas de la uretra posterior, glomerulonefritis aguda, síndrome nefrótico e hipoplasia renal (15).

La ERC es simultáneamente un factor de riesgo y un desencadenante de la enfermedad cardiovascular (ECV) y de otras complicaciones (16-18). En el caso colombiano, el 28\% de la población diabética y entre el 21 y el $36 \%$ de la población hipertensa desarrollan ERC (19), y entre el 54 y el $67 \%$ de las personas con ERC en el país tiene diagnóstico de HTA (20). Los pacientes con ERC en estadios 1 a 4 tienen mayor prevalencia de enfermedad coronaria, falla cardíaca y factores de riesgo cardiovasculares, y además sufren un mayor 
número de eventos cardiovasculares que la población sin enfermedad renal. El porcentaje de pacientes con ERC que mueren por ECV es mayor que el de aquellos que progresan a un estadio de ERC que requiera terapia de reemplazo renal (TRR) (13).

Además de la ECV, los pacientes con ERC tienen mayor probabilidad de desarrollar anemia, alteración de las concentraciones de calcio y fósforo, así como daño en la remodelación ósea. Estos fenómenos aumentan la posibilidad de ocurrencia de fracturas óseas y manifestaciones extraesqueléticas como la calcificación vascular. Durante el curso de la ERC también se puede desencadenar desnutrición energética y proteica, lo que incide en la morbilidad y mortalidad en estos pacientes (5). La prevalencia de estas afectaciones concomitantes es desconocida en el país.

Las cifras de prevalencia, incidencia y mortalidad que se presentan a continuación pueden subestimarse, dados los problemas de subregistro, la comorbilidad con otras enfermedades que enmascaran el diagnóstico y la deficiente vigilancia epidemiológica $(21,22)$.

\section{Prevalencia de la ERC (todos los estadios)}

La prevalencia de la ERC tuvo un aumento significativo, al pasar de 127 pacientes por millón de habitantes (ppmh) en 1993 a 294,6 ppmh en el 2004 (23). Según estimaciones formuladas por Rodríguez en el 2009, el $5 \%$ de la población colombiana tendría ERC (2 160000 personas) (22). En el 2011, más de 788000 personas la padecían, lo que representa el $2 \%$ de la población total (24). En el 2015 se reportaron aproximadamente 962271 personas con ERC; ${ }^{2}$ de ellas, el 15,6\%

2 La CAC reportó 979409 personas con ERC; sin embargo, los cálculos desglosados se refieren a 962271 personas. tenía ERC en estadios 3-5 (517 604). Otras 3385457 personas sufrieron, de forma simultánea con la ERC, enfermedades precursoras como HTA y diabetes (25). En el 2015, los departamentos con mayor prevalencia de ERC en el país fueron el Valle del Cauca, seguido por Caldas y Quindío.

\section{Prevalencia e incidencia de ERC-5}

La prevalencia de ERC-5 en el país ha aumentado de forma progresiva. En el 2008, los casos confirmados fueron 21572 (24); esto es, una tasa aproximada de 53,2 por 100000 habitantes. En el 2011, la prevalencia fue de 57,1 por cada 100000 habitantes (21); es decir, que más de 25000 pacientes renales en el país se encontraban en estadio 5 y requerían TRR (24). En el 2012, se reportaron 27637 casos confirmados (24). En el 2013, el número y la prevalencia se estimó en 65,9 por 100000 habitantes (28 880). La prevalencia de ERC-5 reportada fue mayor en el Valle del Cauca y Quindío $(97 \times 100$ 000), seguidos de Bogotá y Antioquia, y predominó en el régimen contributivo (20). En el 2015 fue de 66,8 por 100000 habitantes (25). Según la cuenta de alto costo (CAC), en el 2013 la tasa de incidencia de ERC-5 fue de 6,5 por 100000 habitantes. Esta tasa fue casi del doble en departamentos como Arauca y Sucre (20). En dos años, la tasa de incidencia fue casi duplicada, de tal manera que en el 2015 fue de 12,3 por cada 100000 habitantes (25).

El aumento de la ERC-5 no es un fenómeno exclusivo de Colombia. Algunos estudios mostraron en Estados Unidos y Canadá desde comienzos de la década del 2000 un crecimiento de la incidencia anual de ERC-5, incluso por encima de la incidencia de ERC (26), lo que implica que los pacientes son captados predominantemente en las fases tardías de la enfermedad. La prevalencia de ERC-5 en Colombia está por encima de países 
como Canadá, Estados Unidos, México y Malasia (25).

\section{Mortalidad por ERC}

Las tasas de mortalidad por ERC no se conocen con exactitud y las cifras oficiales varían dependiendo de la fuente. Según reportes de la CAC, la tasa de mortalidad ajustada de pacientes con ERC en el 2013 fue de 26,9 por 100000 habitantes (excluidas fuerzas militares y policía). Esta varió de manera importante por departamentos. Los que más mortalidad exhibieron fueron Bolívar $(65,6$ $\times 100000)$ y Antioquia $(52,7 \times 100000)$. En el 2015 la tasa de mortalidad alcanzó el 35,6 por cada 100000 habitantes (25).

Específicamente en los casos de ERC-5, la tasa de mortalidad en el 2013 fue de 8,01 $\times 100$ 000, y fue mayor en Guaviare y Caquetá (20). Las muertes reportadas en pacientes que recibían TRR en ese año fueron del $11 \%$. Según reportes científicos, la mortalidad por ECV en pacientes en diálisis es de diez a treinta veces mayor que en la población general. Estas tasas alcanzan el 60 y el $90 \%$ en pacientes en TRR que han sufrido un infarto de miocardio $(5,27)$.

\section{Población en TRR}

Las cifras del sistema no garantizan registros exactos de la proporción de personas que reciben TRR; sin embargo, se estima un aumento debido a la progresión de la ERC a su fase terminal (28). Según Rodríguez, la TRR alcanzó un crecimiento anual del $300 \%$ en la última década. El autor atribuye dicho crecimiento al envejecimiento poblacional, simultáneo con un mayor conocimiento de la enfermedad y sus factores de riesgo y mayor cobertura en el sGSss (22). En el 2010, la CAC reportó 19549 pacientes en TRR diferentes al trasplante, $13384(68 \%)$ en hemodiálisis y $6165(32 \%)$ en diálisis peritoneal (29).

Como se indicó anteriormente, la prevalencia de ERC-5 en el 2015 fue de 66,8 por 100000 habitantes, correspondiente a 28589 personas que, por definición, requieren TRR; sin embargo, según reportes de la CAC, 4560 personas con ERC-5 (16\%) estuvieron sin TRR durante el periodo. Las razones de este fenómeno son desconocidas y su comprensión requiere estudios científicos posteriores. En la tabla 1 se muestra el número total de personas con ERC por estadios, con y sin TRR en el último año.

Tabla 1. Personas con erC CON y SIN trR (2015)

\begin{tabular}{|l|c|c|c|}
\hline Estadio & $\begin{array}{c}\text { ERC con } \\
\text { TRR }\end{array}$ & $\begin{array}{c}\text { ERC } \text { sin } \\
\text { TRR }\end{array}$ & Total \\
\hline Estadio 1 & 864 & & 864 \\
\hline Estadio 2 & 2493 & 441310 & 443803 \\
\hline Estadio 3 & 2165 & 438995 & 441160 \\
\hline Estadio 4 & 1293 & 46562 & 47855 \\
\hline Estadio 5 & 24029 & 4560 & 28589 \\
\hline Total & 30844 & 931427 & 962271 \\
\hline
\end{tabular}

Fuente: CAC, modificada por la autora

Aunque el número de trasplantes ha aumentado en los últimos años, aún se muestra reducido con relación a las necesidades en salud. Esto ocurre tanto por la baja tasa de donantes vivos y cadavéricos (10-16 por millones de habitantes), que cubren solo el $3,7 \%$ de los pacientes en diálisis, como por el bajo número de centros de trasplantes (en el 2009 existían veintiún centros) y su concentración en Bogotá (22).

El preocupante aumento de la incidencia y la prevalencia pudiera explicarse por el entramado de complejos fenómenos relacionados con la globalización, la industrialización y sus consecuentes cambios en los modos y estilos de vida. Así, en el país como en el resto del mundo se han introducido cambios en los 
procesos de urbanización, el aumento en la producción y el consumo de alimentos preelaborados, así como el acceso diferencial a los fármacos, los servicios de salud y otras tecnologías sanitarias de acuerdo con diferentes segmentos poblacionales (30). Por su parte, los cambios en los estilos y modos de vida de la población inducen estrés y aumentan el sedentarismo y el tabaquismo, con lo cual se aumentan los factores de riesgo para desarrollar ERC y sus precursoras, tales como la HTA, la diabetes y la uremia (5,31). Otros factores que inciden en el aumento de la ERC son comportamientos de riesgo como la consulta tardía y la autoformulación de medicamentos con potencial nefrotóxico (22).

\section{Condiciones de equidad social y sanitaria en la presentación y atención de servicios de salud}

Ya se ha esbozado que en el país persisten altas tasas de incidencia, prevalencia y mortalidad por ERC; sin embargo, la distribución no es homogénea entre los distintos segmentos poblacionales. Tanto los determinantes sociales del desarrollo de ERC como la forma de atención se producen de manera diferencial entre los distintos grupos sociales; esto es así no solo en Colombia, sino en el mundo. Globalmente, las personas más susceptibles de adquirir la ERC tienen condiciones estructurales asimétricas relacionadas con sus modos de vida. Estos diferenciales implican no solo una condición de vulnerabilidad diferente para enfermar por ERC, sino que las personas afectadas también soportan conductas de inequidad en la prestación de servicios de salud y el acceso a los tratamientos (32-34). Ambos tipos de diferenciales se presentan en virtud de la raza, la zona geográfica y la condición social $(6,32,33)$.
Las desigualdades en salud en el caso de la ERC representan inequidades porque, al tratarse principalmente de problemas de salud crónicos, con frecuencia están asociados con deficiente manejo por parte del sistema de salud, lo que, por lo general, afecta a menudo a los grupos con menor acceso a los servicios de salud y menor calidad en ellos (35). Se ha informado que las personas en edad productiva, las mujeres, los mayores de sesenta años y las personas más pobres tienen mayores dificultades para acceder al sistema de salud y, por lo tanto, padecen discriminación y exclusión. Estos diferenciales, por ser injustos e inaceptables, revelan inequidades sociales y sanitarias (36-38). Para ilustrar estas inequidades puede citarse el estudio de Martínez, en el cual se incluyeron pacientes diabéticos que padecieron ERC-5. Este estudio encontró que los pacientes pertenecían predominantemente a grupos con bajo nivel educativo y bajo estrato socioeconómico. Adicionalmente, se encontraron diferencias en la calidad de la atención en las poblaciones del régimen subsidiado (19). Algunas investigaciones en el ámbito mundial refuerzan este hallazgo, en la medida en que han encontrado asociación entre la ERC y el bajo nivel de información en salud (39).

\section{La ERC y la respuesta por parte del sistema de salud}

Además de las condiciones de vida, el manejo de la ERC está condicionado en cualquier país por la forma de organización y el funcionamiento del sistema de prestación de servicios de salud. En el Sistema General de Seguridad Social (sGSss) se incluyó explícitamente, a partir de la Ley 100 de 1993 (40) y de su marco normativo complementario, el manejo clínico de esta enfermedad, puesto que en el anterior Sistema Nacional de Salud (sNs) ello no se contempló. Desde la promulgación de la mencionada Ley 100, los pacientes con 
EAC son atendidos por su aseguradora, de acuerdo con el régimen de afiliación al que pertenezcan. Estos servicios están cubiertos por el Plan Obligatorio de Salud (POS) y, por lo tanto, históricamente se manejaron de manera diferencial entre los dos regímenes de afiliación (régimen contributivo y régimen subsidiado) (41). En normas más recientes promovidas por la Sentencia T-760 del 2008 de la Corte Constitucional se exigió la homologación de un mismo paquete de servicios para los dos regímenes (42). Con la Ley 1438 del 2014 (43), el Gobierno Nacional intentó responder a este lineamiento mediante la unificación del pos $(43,44)$; sin embargo, se desconoce hasta el momento el impacto de la norma en la cobertura y la calidad de la atención para estos pacientes.

\section{Guías de atención clínica y actividades de prevención}

En el desarrollo del sGsss se introdujeron guías clínicas de manejo específicas para la atención individual de la ERC $(5,27)$, guías de promoción de la salud y prevención de la enfermedad (45), la definición de las actividades de protección específica, detección temprana y atención de enfermedades de interés en salud pública como la DM y la HTA (46) y las actividades del Plan Decenal de Salud Pública, así como los mecanismos de protección financiera $(5,27,47-53)$. Estos lineamientos son útiles, en la medida en que se basan en estándares biomédicos (basados en la evidencia) que permiten retardar o prevenir la progresión de la enfermedad $(18,54)$, pero no son vinculantes y, por lo tanto, en el país no existen programas universales de renoprotección.

Su aplicación es un proceso complejo que requiere integralidad e integración de los servicios de salud; es decir, que involucra el diagnóstico oportuno; el manejo de enfermedades concomitantes, de las complicaciones y de sus factores de riesgo (prevención secundaria); el monitoreo de la severidad y la progresión de la enfermedad en el tiempo; el monitoreo y control de su estado nutricional y el suministro de información al paciente sobre su enfermedad y los estilos de vida que reducen los riesgos de progresión. Por último, el adecuado manejo implica la oportuna y correcta referencia al nefrólogo (27). En estas guías, la prevención primaria para reducir los factores de riesgo asociados con la enfermedad es marginal (5).

El manejo de enfermedades concomitantes y de las complicaciones incluye, entre otros, la evaluación y el control de la enfermedad cardiovascular, la diabetes, la anemia y otros desórdenes metabólicos y estructurales. Específicamente, se requiere efectuar el monitoreo y control de la presión arterial y la dislipidemia, así como de los niveles de calcio, fósforo, hormonas tiroideas, hemoglobina, glicemia y proteinuria. Es necesario también evitar y corregir las causas del deterioro agudo de la TFG, como la hipovolemia y la obstrucción del tracto urinario (5). El monitoreo de la severidad y la progresión de la enfermedad en el tiempo exige identificar si la ERC es estable, lentamente progresiva, aguda, subaguda o si presenta agudización. Por otra parte, es preciso evitar la iatrogenia, mediante la vigilancia de fármacos como los antiinflamatorios no esteroides (AINE), los antidiabéticos de eliminación renal, los fármacos retenedores de potasio, los agentes nefrotóxicos (aminoglucósidos y anfotericina b) y los medios de contrastes radiológicos.

Otras actividades de prevención secundaria involucran la evaluación habitual del estado nutricional, la ingesta proteica y calórica, el control dietario de proteínas, fósforo y calcio, la suplementación con aminoácidos esenciales, el control de la anemia y el manejo de la obesidad. De forma adicional, se recomienda brindar a los pacientes asesoría 
sobre estilos de vida saludable relacionados con la suspensión del tabaquismo, el mantenimiento del peso y la práctica del ejercicio.

La remisión oportuna a nefrología se debe realizar con base en la severidad o estadio de la ERC, la velocidad de progresión (incremento rápido de la creatinina sérica) y las complicaciones y otras características del paciente, como son la edad avanzada y otras enfermedades concomitantes o sistémicas (13). En la guía clínica colombiana se recomienda la remisión al nefrólogo a todo paciente en estadios 3, 4 y 5 (cuando la $\mathrm{TFG}<60 \mathrm{~mL} / \mathrm{min} / \mathrm{m}^{2}$ ), pero se refiere a una remisión obligatoria cuando la TFG sea inferior a $30 \mathrm{~mL} / \mathrm{min} / \mathrm{m}^{2}$ y cuando en dos exámenes consecutivos la creatinina sérica sea de $2 \mathrm{mg} / \mathrm{dL}$ en los hombres y $1,7 \mathrm{mg} / \mathrm{dL}$ en las mujeres (5,27). Algunos autores también recomiendan la remisión en aquellos casos cuyo control de la creatinina sérica indique incrementos de $0,5 \mathrm{mg} / \mathrm{dl}$ cada dos a tres meses o de $1 \mathrm{mg} / \mathrm{dL}$ en un mes (13).

En los casos de ERC-4 y -5, el nefrólogo debe discutir con el paciente la modalidad de terapia de reemplazo renal, efectuar el acceso a diálisis e indicar las vacunas necesarias que amerita el manejo específico. El tipo de diálisis depende en gran parte de la preferencia y las condiciones del paciente. En el paciente con ERC que requiere iniciar diálisis y tiene función renal residual, la primera opción de tratamiento es la diálisis peritoneal. Esta se puede mantener durante el tiempo en que el paciente tenga función renal residual (FRR), pero una vez pierda esta función o desarrolle compromiso cardiovascular se debe continuar con hemodiálisis. La dosis estándar de hemodiálisis es de tres sesiones a la semana durante cuatro horas por sesión, pero el tiempo o la frecuencia cambian en pacientes con problemas cardiovasculares o inestabilidad hemodinámica. Estos pacientes requieren anticoagulación, prevención y tra- tamiento de infecciones como la tuberculosis ( $\mathrm{TBC})$, la hepatitis B (HBV), la hepatitis C ( HCV) y el viH. También es necesario proveer vacunación contra diferentes agentes como la difteria, el tétanos y el neumococo. Por último, todo paciente con falla renal crónica terminal debe ser considerado potencial receptor de un trasplante renal $(5,27)$. Las recomendaciones detalladas para el manejo específico de pacientes con y sin otras comorbilidades, el ingreso a diálisis (hemodiálisis o diálisis peritoneal) en pacientes con ERC-5 y las recomendaciones del cuidado paliativo para pacientes con ERC-5 sin indicación de diálisis pueden encontrarse en la CAC del 2013 y en la guía de atención clínica $(5,55)$.

Pese al amplio y complejo marco normativo del país para el manejo de la ERC y a la expedición de guías clínicas y de prevención, la respuesta del sector salud no ha sido eficaz. Esto puede evidenciarse con los datos epidemiológicos mostrados anteriormente. Varios factores podrían explicar parcialmente dicha ineficacia: problemas relacionados con la oportunidad y calidad en el diagnóstico y el tratamiento, ambigüedad en la clasificación del "alto costo", deficiente gestión del riesgo, alcance limitado en las acciones de salud pública, barreras administrativas y estructurales del sistema de salud, altos costos e ineficiencia en la distribución de recursos y pérdida del papel rector del Estado. A continuación se explican estos factores en detalle.

\section{Modelo de atención y ausencia de implementación}

El modelo de prevención y control de la ERC denominado "Componente de un modelo de salud renal" fue propuesto por Fedesalud, en concertación con diferentes actores sociales, y fue aprobado por el Ministerio de Salud y Protección Social. Este modelo incluye programas de prevención primaria y secundaria y acepta, teóricamente, que 
para la implementación se debe disponer de los recursos necesarios para satisfacer las necesidades médicas, emocionales, sociales, nutricionales y económicas de los pacientes.

En cuanto a la prevención primaria, sugiere el desarrollo de acciones orientadas al control de los factores de riesgo ocupacionales causados por la exposición a sustancias nefrotóxicas y al control sanitario de sustancias tóxicas en el medio ambiente. Con relación a la prevención secundaria, propone el manejo de las enfermedades precursoras, el diagnóstico temprano, la aplicación de medidas de nefroprotección y el aumento de los tiempos de progresión para retardar la entrada a la diálisis. Para lograrlo, se propuso el tratamiento integral y adecuado a las condiciones de los pacientes, así como la atención de las principales patologías causantes de la ERC. En los casos de sustitución renal, se recomienda optimizar el tratamiento para disminuir los costos de hospitalización, las complicaciones, la incapacidad y la mortalidad. Para ello se plantea el inicio oportuno de la terapia de reemplazo renal, incluido el trasplante preventivo cuando fuese posible.

El modelo propone el empoderamiento y la educación de los pacientes, familiares y cuidadores sobre el riesgo, la vulnerabilidad y los comportamientos protectores. Otra recomendación del modelo incluye el apoyo social expresado así: "seguimiento activo de la persona, el apoyo social y la rehabilitación integral cuando es requerida, además de las acciones asociadas con el proceso de desarrollo social".

Para cumplir con el modelo se especifican las acciones administrativas y operativas, delegadas en las entidades administradoras de planes de beneficio (EAPB) y las IPS, respectivamente, y se identifican las características de operación de registro y sistema de información, la conformación del equipo de trabajo básico con profesionales de medicina, enfermería, psicología, nutrición y trabajo social, entre otros, designando a cada uno los procesos particulares en los que deben participar. También se definen las capacidades y calidades requeridas en la red de laboratorios, el funcionamiento de la red de servicios, las características del sistema de referencia y contrarreferencia y la movilidad de los pacientes, así como procesos administrativos de aseguramiento de la calidad.

En materia de salud pública, la autoridad sanitaria ha expedido orientaciones para la planificación y ejecución de actividades colectivas cuya finalidad es la reducción de los riesgos y el logro de cambios en el comportamiento hacia estilos de vida saludables. Las estrategias planteadas para el logro de estos objetivos se han centrado básicamente en la promoción de la actividad física y la información (56), aspectos importantes pero no suficientes para el control de la enfermedad, especialmente si se reconoce que está asociada con factores de riesgo proximales, pero que en particular se relaciona con fenómenos de carácter estructural, como se indicó previamente.

No obstante lo anterior, en la práctica los programas son sobre todo asistenciales, con énfasis en la diálisis (con coberturas no universales), pero relega los programas de prevención primaria y gestión del riesgo en pacientes en estadios tempranos y con enfermedades precursoras (22). El enfoque de manejo ha sido predominantemente individual y biomédico, pero no alcanza a aportar una respuesta adecuada para mitigar el problema o la resolución de las complicaciones clínicas y sociales derivadas.

\section{Barreras administrativas y estructurales del sistema de salud}

Aunque se conocen pocos estudios de las condiciones de atención específicamente 
para las poblaciones con ERC, son múltiples los estudios que en Colombia develan las importantes limitaciones estructurales y organizativas del sistema de salud para la población general y las poblaciones vulnerables. Se ha demostrado que las barreras de acceso a los servicios de salud impiden la atención con calidad, limitan la protección clínica y financiera de los afectados (43) e incrementan la inequidad social y sanitaria $(57,58)$. Algunos informes recientes han mostrado la persistencia de las desigualdades entre los distintos grupos poblacionales (44). En el caso del sistema de salud colombiano, la inequidad se evidencia en razón de que existen diferentes regímenes de afiliación y que se prestan servicios diferenciados a la población según su capacidad de pago; por lo tanto, terminan estableciendo una brecha entre la población según su condición socioeconómica (44).

En el caso de la ERC se desconocen el acceso, la utilización y la continuidad de los servicios de salud en Colombia. Sin embargo, algunos datos internacionales han informado acerca de las barreras financieras para el acceso a los servicios. Para ilustrar la situación se puede citar el análisis de Gotsadze, quien encontró que cerca del $40 \%$ de la población con alguna EAC no acude a los servicios de salud (6). Adicionalmente, quienes acceden al servicio no siempre continúan o finalizan su tratamiento (59).

Además de los problemas financieros y administrativos, existen problemas estructurales en el sistema de salud relacionados con la maximización de la rentabilidad y la competencia que se evidencian en problemas de calidad del servicio prestado: en el caso de la ERC, se ha develado que se pueden prestar servicios con criterios de optimización de la operación para lograr ventajas competitivas entre prestadores, lo que compromete los estándares de calidad (32).

\section{Ambigüedad en la clasificación del "alto costo" y la gestión del riesgo}

A partir del Decreto 5261 de 1994 (41) se definieron los tratamientos para las EAC; sin embargo, la definición misma constituye uno de los problemas para el manejo eficaz de la ERC en el SGSSS, debido a que no se propone una clasificación en virtud de la enfermedad ni del enfermo, sino de los tratamientos para los estadios más avanzados. Esta ambigüedad en lo que se considera el "alto costo" ocasiona problemas en la atención y en los procesos administrativos que implican pagos a terceros. De acuerdo con la Resolución 2565 (47), solo se consideran de alto costo las personas que están en estadio 5 de la ERC con necesidad de TRR. Esto supone que, primero, la atención general no relacionada con estas terapias sustitutivas (tratamientos explícitamente definidos en la norma) no deja lugar a reajustes financieros $\mathrm{y}$, por lo tanto, se desincentiva la prevención y el tratamiento oportuno. Esta concepción impide que se realicen acciones continuas y permanentes para evitar la progresión y, por lo tanto, los sujetos pueden presentar problemas biomédicos evitables y sobreagregados causados por la inoportunidad del tratamiento. Por otra parte, no todos los pacientes tienen garantizado el acceso, debido a barreras financieras en el momento de acudir al sistema de salud, puesto que solo las personas en estadio 5 estarían exentas de copagos, según la norma $(60,61)$.

Desde el punto de vista administrativo, la ambigüedad en la definición de lo que significa el alto costo produce dificultades para financiar la atención por causa de los recobros, para definir los montos correspondientes al reaseguro establecido por ley para las enfermedades de alto costo y lograr con éxito las reclamaciones correspondientes (62). Debido a esta situación y a los tipos de contratación permitidos, las IPS no realizan 
el seguimiento adecuado, según los criterios definidos en las guías de atención integral, a los pacientes para evitar las glosas. Estas situaciones en la atención producen un círculo vicioso que va en contra de la seguridad del paciente, su calidad de vida y su bienestar.

\section{Altos costos e ineficiencia en la distribución de recursos}

En Colombia no se cuenta con datos sistemáticos nacionales sobre costos asociados con las EAC y, en particular, para la ERC, toda vez que el Ministerio de Salud y Protección Social, entidad rectora del sistema de salud, delegó la función de la vigilancia en salud pública y de la gestión financiera a la cuenta de alto costo. Sin embargo, se sabe que la ERC ha producido una escalada de costos que podría poner en riesgo financiero al sistema, principalmente bajo el modelo de atención que se privilegia hoy. El tratamiento y los medicamentos de la ERC - sobre todo en estadios avanzados- imponen altos y crecientes costos económicos para la sociedad y el sistema de salud, puesto que exigen personal calificado y tecnología sofisticada y costosa (63). Según el Acuerdo 217 del 2001, se ha considerado la ERC en la etapa de hemodiálisis renal y diálisis peritoneal como aquella que tiene un mayor impacto financiero y una mayor desviación del perfil epidemiológico (64).

Con relación a los gastos en salud, en Colombia se ha estimado que las EAC absorben aproximadamente el $10 \%$ de la UPC, que es diferencial para los regímenes contributivo $(7,29 \%)$ y subsidiado $(14,66 \%)$. No se sabe con claridad por qué ocurre esto, toda vez que por norma estas enfermedades deben ser reaseguradas (58). En el 2005, un estudio encontró que los costos por eventos catastróficos como porcentaje del ingreso operacional fueron mayores en el régimen contributivo. Esta situación fue explicada por el mejor conocimiento de derechos y coberturas por parte de los actores del sistema en el régimen contributivo y su consecuente proceso de remisión a niveles superiores, la posible "resolución progresiva de la carga de enfermedad acumulada por esta patología y la dilución del riesgo por el volumen alcanzado de usuarios", junto con la afiliación selectiva y la utilización de servicios de mayor nivel de complejidad generados por las deficiencias en la calidad de servicios (65).

En el 2004, el costo de las diálisis alcanzó los $\$ 450000$ millones de pesos, y se calcula que en los últimos años esta ha consumido entre el 2 y el $4 \%$ del gasto en salud en el país y seguridad social en salud, respectivamente $(19,23)$. Los costos de trasplante varían según el donante y son significativamente altos cuando se introducen terapias para controlar el rechazo (8). En el 2009 se estimó para el país un costo superior a 48000 millones de pesos por diálisis y de 37500 millones por trasplantes (22). ${ }^{3}$ Desde una perspectiva institucional, esta enfermedad también consume un alto porcentaje del gasto. Así, un estudio en una IPS mostró que la ERC representó el $19 \%$ de la carga financiera entre 1993 y 2003 (57).

El país gasta ingentes cantidades de recursos, a pesar de los escasos resultados evidenciados. Según la CAC, en el 2015 se distribuyeron 65000 millones (US\$26 millones de dólares, aproximadamente) entre cincuenta aseguradoras. De acuerdo con esta fuente, en cinco años se logró la disminución del costo del tratamiento en la terapia dialítica por pa-

3 No se cuenta con datos más actualizados de los costos que se generan por la atención de la ERC en Colombia. Estos fueron solicitados tanto a la CAC como al Ministerio de Salud y Protección Social en el marco de la investigación, pero no se obtuvo una respuesta favorable. En comunicación personal con la CAC del año 2015 se indicó únicamente que el costo de la diálisis era de aproximadamente $\$ 2400000$ pesos colombianos. 
ciente, con un ahorro para el sistema de salud de $\$ 900000$ millones de pesos, y se evitó el ingreso de aproximadamente 5169 pacientes a TRR, lo cual, según esta fuente, representó un ahorro aproximado de $\$ 600000$ millones de pesos (66).

Ante las inconsistencias mostradas entre la epidemiología y el gasto, surgen varias inquietudes: ¿cuántos recursos puede ahorrar el sistema si, en lugar de centrar los incentivos en las etapas más avanzadas de la enfermedad, se concentraran los esfuerzos en mejorar los modos de vida de la población? Si se invirtieran los recursos en mecanismos eficaces de prevención de la enfermedad, quizás los "ahorros" para el sistema de salud quedarían acompasados de atención con calidad y equidad sanitaria. ¿Serán los enunciados ahorros simples mecanismos de contención de costos? ¿Corresponden los casos de TRR evitada a logros en el mejoramiento de la atención y conducen al mejoramiento de la calidad de vida o podrían estar contribuyendo a las tasas de mortalidad? Si bien no existe un acervo de investigaciones que muestren esta asociación, datos de un estudio publicado por Sanabria en el 2015, con información retrospectiva de quinientos pacientes, mostró que la tasa de mortalidad fue mayor que la tasa de inicio de diálisis (2,9\%, frente a $2,1 \%$ de pacientes/año) (67).

\section{Dificultades del talento humano para realizar el diagnóstico y tratamiento oportuno}

La ERC se diagnostica y se trata usualmente de manera tardía, debido a que es una enfermedad de difícil diagnóstico y tratamiento, por cuanto los síntomas suelen ser inespecíficos y silenciosos y, a menudo, pueden solaparse con otras afecciones, pero también debido a problemas de talento humano que impiden un manejo oportuno. Algunos autores indican que existe insuficiente formación de los médicos respecto a los criterios de clasificación y definición de la enfermedad, de identificación de los factores de riesgo o enfermedades precursoras y de la adecuada remisión al nefrólogo $(22,68,69)$. Esta situación podría explicarse parcialmente por limitaciones en la formación básica en el área específica, por la emergencia de escuelas de medicina y por las restricciones en las prácticas clínicas (22). Por otra parte, existe un bajo número de especialistas formados en nefrología y no se ha caracterizado claramente la necesidad de formación para responder a las necesidades del país. En el 2009 se calculaba que en el país existían alrededor de 220 nefrólogos, distribuidos en 140 unidades renales, y solo operaban ocho programas de posgrado, pero concentrados en tres ciudades del país (22). A la fecha no se cuenta con información actualizada que permita analizar la evolución de la situación y generar políticas públicas que respondan a las necesidades concretas. Como consecuencia de ello (pero no exclusivamente), las enfermedades crónicas como la ERC son detectadas cuando los pacientes se encuentran en estado crítico; es decir, cuando ya requieren tratamientos sustitutivos complejos y costosos que implican terapias de reemplazo renal como la diálisis, la hemodiálisis y el trasplante (32). Una vez diagnosticada, existen limitaciones para efectuar una adecuada prevención secundaria y terciaria que evite la progresión de la enfermedad y las complicaciones (22). Esto supone, además de pérdidas en vidas, un gasto médico mayor para los pacientes y para el sistema de salud.

\section{Deficiencias en la vigilancia y monitoreo de los resultados en salud}

De acuerdo con los objetivos de nefroprotección propuestos en las guías de atención clínica, en el modelo de atención y en los planes de salud pública, se han determinado los indicadores de gestión, de procesos y de resultados en salud y, en particular, aquellos 
relacionados con la ERC y enfermedades precursoras, la diálisis peritoneal, la hemodiálisis y el trasplante en adultos y niños (70-73). Además de esto, mediante la Resolución 4700 del 2008 (74) se determinó que las EPS y las demás entidades obligadas a compensar deben reportar información detallada de los pacientes con ERC y de los pacientes con patologías precursoras a la CAC, de manera que se pueda monitorear la evolución de dichos pacientes y la progresión de la enfermedad. Esta información sirve además como apoyo para distribuir los recursos según lo estipulado en la Resolución 3215 del 2007 (75).

A pesar de este laborioso trabajo de identificación de indicadores básicos para el seguimiento de los programas, la evidencia del monitoreo - según información reportada en las páginas oficiales de la CAC y de la autoridad sanitaria - es muy limitada. Algunos informes de la situación de la ERC muestran tasas elevadas de enfermedades precursoras y un muy bajo porcentaje de personas en seguimiento. Los indicadores presentados por la CAC sobre los resultados de los pacientes en diálisis por IPS presentan varios inconvenientes: el primero de ellos está relacionado con el bajo número de indicadores evaluados: dos de ellos orientados a la evaluación de proceso (pacientes en hemodiálisis con catéter y dosis de la diálisis) y tres de resultados que miden solo seguimiento de tres analitos (hemoglobina, fósforo y albúmina); en segundo lugar, el informe concibe como indicadores estándares aquellos cuyo cumplimiento oscila solo entre 40 y $80 \%$ de la actividad; valores por debajo de este cubrimiento se consideran bajos o medios. Adicionalmente, muestra que el cumplimiento de las actividades mínimas propuestas puede ser bajo o moderado, dependiendo de la IPs que presta el servicio, pero no se identifican las sanciones o estrategias de mejoramiento que permitan avanzar hacia el cumplimiento de las metas
(76). A pesar de estar indicado, en el país la captación de estándares básicos como HTA y DM es de solo 38 y $65 \%$, respectivamente (20).

En las bases de datos bibliográficas se encontraron muy pocos estudios que intenten determinar los procesos o resultados en salud de los programas; entre ellos, uno que determinó adherencia de los pacientes con IRC a las citas programadas. Este estudio encontró una adherencia de 98,6\% (1939/1967), cifra que fue explicada por la conformidad con el horario y el personal de salud, así como por información suministrada a los pacientes sobre la importancia que tiene la adherencia en la eficacia del tratamiento (77). Por el contrario, otro estudio encontró que la adherencia al tratamiento no farmacológico en pacientes con hipertensión y diabetes en servicios de baja complejidad fue de $9,4 \%$; también evidenció que la actividad física estuvo ausente en $75 \%$ de los pacientes, que más del $19 \%$ de la población consumía sal frecuentemente y que entre los diabéticos el $18 \%$ consumía carbohidratos con frecuencia (78).

Un estudio reciente realizado en Antioquia, en el que se incluyeron 2317 pacientes con ERC-5, mostró que solo un porcentaje cercano al 10\% recibió atención psicosocial, nutricional o por equipos interdisciplinarios (79). Un análisis sistemático de las investigaciones en Colombia dirigidas a indagar sobre la calidad de la atención de la diabetes — una de las enfermedades precursoras más importantes-encontró fallas relacionadas con el tipo de profesional que atendía la enfermedad, problemas en la provisión de medicamentos al paciente: personas no tratadas para otras enfermedades asociadas; porcentajes variables de control de glicemia, perfil lipídico y tensión arterial; fallas en el cumplimiento de metas; baja información con relación a los cuidados no farmacológicos y bajas coberturas en la prescripción de insulina y gastos de bolsillo (80). 
Un estudio en Ibagué encontró que de los 74 pacientes estudiados, el 93,5\% inició hemodiálisis en forma no programada (81). El estudio de Martínez indagó por la calidad de la atención para la población en diálisis atendida en varias clínicas de dos ciudades del país y encontró que, según algunos estándares, el 75\% de la población estudiada obtuvo una calidad de atención deficiente y ausente, y que esta calidad era diferencial de acuerdo con el régimen de atención (19). El régimen de afiliación es un indicador indirecto de la condición socioeconómica y, por tanto, un proxy de las inequidades sociales. Otras investigaciones han mostrado que la adherencia a los tratamientos farmacológicos es baja y que difiere entre los diferentes subgrupos poblacionales (82).

En el país tampoco se cuenta con información concluyente acerca de las capacidades de los pacientes o sus familiares para realizar la diálisis en casa con la seguridad necesaria. Al respecto, hay un estudio realizado en Bogotá que identificó la capacidad de autocuidado de los pacientes en diálisis, mediante pruebas estandarizadas, según el cual se determinó que el 77,4\% de los participantes quedaba clasificado en el rango de operatividad de la capacidad de agencia de autocuidado alta. Este estudio encontró que la mitad de los participantes practicaba ejercicio y el 81,4\% mencionó seguir la dieta indicada, pero el $61 \%$ no fue adherente al tratamiento (83).

Desde la introducción de la Ley 1438 que modifica el sGsss (43), se plantea la evaluación de la calidad de la atención en salud basada en resultados. Pese a que han transcurrido cinco años a partir de la sanción de la ley, no existen informes o investigaciones sobre la gestión de las aseguradoras, así como tampoco de la relación entre la calidad de la atención con los procesos de contratación que se llevan a cabo entre las EPS y las IPS.
Este tema resulta prioritario, toda vez que la información de la Asociación Colombiana de Nefrología indica que las EPs se ocupan más de asegurar la financiación de los pacientes que de la gestión integral del riesgo. Asimismo, señala que la disminución de las tarifas pagadas a las IPS por la atención de los pacientes con ERC puede comprometer la calidad del servicio o presionar a los prestadores para incluir un mayor número de servicios o medicamentos dentro del mismo paquete pagado, incluso aquellos no incluidos en el Pos, que por norma pueden ser recobrados al Fosyga (22).

No existe evidencia acerca de que la auditoría sobre la gestión del riesgo mejore los procesos de calidad, así como tampoco de las sanciones o restricciones del asegurador o de su red prestadora de servicios para participar en la distribución de los recursos del sistema o en los contratos para continuar atendiendo a los pacientes cuando los resultados de gestión, procesos o resultados en salud son insatisfactorios. De lo que sí existe una amplia evidencia es que la subutilización de cuidados estándares puede contribuir al aumento de la morbimortalidad (84).

\section{El papel de la autoridad sanitaria}

La escalada de costos para el manejo de la ERC ha estado acompañada de la permisividad por parte del gobierno que promueve incentivos financieros a la atención por alto costo y desestimula la prevención primaria. A partir del 2007 y el 2008, las aseguradoras lograron que se hiciera un reajuste a la UPC para "compensar los desequilibrios" en la prestación de los servicios a los pacientes con estas enfermedades, de tal forma que el incentivo se genera con el reporte del número de casos con pacientes en diálisis o TRR. Por otro lado, los excedentes del Plan Nacional de Salud Pública fueron dirigidos al financiamiento de las acciones de "diagnós- 
tico temprano y reducción de la nefropatía diabética e hipertensiva" ejecutadas por las EPS (85), dineros que por ley corresponden a las acciones de salud pública, pero que fueron reasignados a las aseguradoras para cumplir con una función ya delegada y pagada según las directrices de la Ley 100 de 1993 y normas complementarias $(62,86)$. La norma en mención permitió que el giro y el desembolso se realizaran a las diferentes entidades, sin que fuera necesaria su administración a través de la subcuenta de eventos catastróficos; es decir, que esos recursos pasarían a ser administrados directamente por las EPS. Estos hechos sugieren que, además de un alto gasto relacionado con las características de la enfermedad, el incentivo financiero ha estado centrado en la atención de la enfermedad en estados avanzados y en la compensación extra a las EPs por cumplir su misión dentro del sistema.

Pese a las prebendas logradas, las EPs han argumentado que las EAC son responsables de los desequilibrios financieros del sistema de salud (87) y han sido señaladas por el desfinanciamiento, especialmente en el régimen subsidiado en salud, debido a la diferencia entre los costos de atención y la unidad de pago por capitación subsidiado (UPC-S). Pero mientras prevalezca el modelo que incentiva los tratamientos de alto costo, los recursos serán insuficientes para la financiación del sistema.

Es importante mencionar que las EAC demandan no solo tratamientos médicos de largo término, sino también procesos que conllevan frecuente hospitalización y consultas por urgencias $(57,88)$. En Colombia, a pesar de que las EAC son cubiertas por el sGsss, algunos estudios han mostrado que la protección financiera se da especialmente en los casos de consulta externa y medicamentos, pero menos en los eventos de hospitalización, lo que aumenta el gasto de bolsillo como principal fuente de financiamiento e impone múltiples retos sobre las condiciones de vida de los afectados $(89,90)$.

Por otra parte, las aseguradoras cambian a los pacientes entre las distintas IPS, de acuerdo con las tarifas ofrecidas, y no necesariamente con criterios de calidad o de respeto por el principio de la libre escogencia (22). La serie de factores anteriormente descritos va en detrimento de la salud y la calidad de vida de los pacientes, genera mayores pérdidas económicas en la renta nacional y doméstica y, por lo tanto, lleva a quienes padecen dichas enfermedades a estar por debajo del umbral de pobreza (32). Peor aun, los incentivos a la atención de mayor nivel de complejidad, la transferencia indebida de fondos de los programas de salud pública hacia las EPS y la escalada de costos - sin control ni vigilancia por parte de la autoridad sanitaria- son en gran parte responsables de la situación de salud y la crisis por la que atraviesa el sistema de salud.

Con relación a las deficiencias de las políticas públicas para el control de la enfermedad, Rodríguez plantea deficiencias en los procesos de promoción de la salud, puesto que no están dirigidos a incidir sobre las condiciones de vida de la población y sus comportamientos. Por otra parte, cuestiona la falta de compromiso de los actores del sistema para la implementación del modelo de prevención y control propuesto desde el 2006 y señala al Gobierno por su falta de capacidad para el monitoreo, la vigilancia y el control en el sistema, así como recalca la debilidad de la autoridad sanitaria para ejercer mecanismos de prevención que alerten a la población sobre los riesgos de la automedicación y del consumo de medicamentos nefrotóxicos y, del mismo modo, implementar controles efectivos a la venta de los medicamentos (22). 


\section{Algunas conclusiones y recomendaciones}

La situación de salud relacionada con la ERC en el país es compleja, toda vez que la incidencia, la prevalencia y la mortalidad por causa de esta enfermedad van en aumento. Esto implica no solo el desmejoramiento de la calidad de vida de los pacientes, sino también una escalada de costos importante que pone en riesgo financiero al sistema de salud, pese a su carácter prevenible. Ante esta situación, la respuesta del sistema de salud y de la autoridad sanitaria ha sido insuficiente. Las debilidades de la respuesta están relacionadas con fallas en la oportunidad y calidad en el diagnóstico y tratamiento, la ambigüedad en la clasificación del alto costo, la instauración de mecanismos basados en la gestión del riesgo, el limitado alcance de las acciones de salud pública y las barreras administrativas y estructurales del sistema de salud. Estas situaciones concurren con una escalada de costos, la ineficiencia en la distribución de recursos - que incentiva el alto costo en lugar de la prevención-y la pérdida del papel rector del Estado.

La complejidad de la situación de la ERC en un contexto de cambios globales en el mundo contemporáneo amerita instaurar mecanismos de salud pública que prevengan, traten y mitiguen el impacto de esta enfermedad. Ello implica trascender los factores de riesgo individuales, para reconocer la influencia de complejos fenómenos sociales y, por lo tanto, implementar cambios paradigmáticos que ofrezcan una mejor respuesta para su manejo.

La planificación sanitaria debe llevar al mejoramiento de los resultados en salud, entendidos como la reducción de la incidencia, de la mortalidad y de la discapacidad, así como la mitigación del impacto psicosocial y económico que se genera en los pacientes afectados. Para ello, es necesario efectuar un cambio de modelo de atención por uno más comprehensivo e integral, que permita impactar en los determinantes de la salud y los modos de vida, reducir los riesgos y garantizar el manejo integral de la enfermedad como un proceso que amerita oportunidad y calidad.

El mejoramiento de la calidad en la atención integral solo es posible si la autoridad sanitaria formula directrices para el mejoramiento de la gestión de los procesos técnico-científicos, administrativos y financieros de los actores del sistema, de forma obligatoria para todos ellos. Ya se ha recorrido un camino interesante en la expedición de guías clínicas y de prevención; sin embargo, es necesario introducir un sistema de monitoreo que permita garantizar el logro de estándares clínicos y de los resultados en salud. Esto incluye la necesidad de que el Invima ejerza un control efectivo sobre la calidad de los productos farmacéuticos y que mejore los procesos de información a los usuarios relacionada con los riesgos de su uso. Asimismo, es preciso que los organismos territoriales del orden departamental implementen sistemas adecuados y confiables de habilitación a las instituciones de salud y que el Ministerio de Salud y Protección Social fortalezca su capacidad para determinar las condiciones de operación de las EPS y que la Superintendencia de Salud ejerza una adecuada vigilancia, inspección y control para garantizar su cumplimiento. Es necesario efectuar un cambio en el sistema de incentivos hacia la prevención primaria que procure la eficiencia en el manejo de los recursos, la transparencia en el manejo de la información epidemiológica y financiera y la implementación de sistemas de auditoría que impliquen el cumplimiento de estándares obligatorios de calidad en la atención.

Se recomienda además que la autoridad sanitaria implemente sistemas integrados de información que permitan proyectar las necesidades en salud y los requerimientos de 
formación de talento humano, así como la infraestructura y los procesos necesarios para prestar los servicios de salud integrales, y que por supuesto dicten pautas al sector educativo para desarrollar las competencias necesarias.

Es preciso que la autoridad sanitaria determine la obligatoriedad y las condiciones para que las EAPB garanticen las redes de prestación de servicios por niveles de atención $\mathrm{y}$, con enfoque territorial, fortalezcan los niveles de menor complejidad y determinen las rutas para la adecuada referencia y contrarreferencia, para garantizar el derecho a la salud y la prestación de servicios integrales, como se plantea en la Ley 1438, la Ley Estatutaria y la nueva política de atención integral $(43,91,92)$.

\section{Agradecimientos}

Agradezco a Yina Berona, Tania Cardona, Marcela Ortiz y Erika Alejandra Ríos, quienes participaron como miembros del semillero de investigación en Sistemas de Salud (Sisalud) de la Facultad Nacional de Salud Pública en la búsqueda y sistematización bibliográfica.

\section{Financiamiento}

Este trabajo fue financiado por el Fondo de Apoyo Docente del Centro de Investigación de la Facultad Nacional de Salud Pública y por la Estrategia de sostenibilidad de Comité para el Desarrollo de la Investigación (CODI) de la Universidad de Antioquia (2012-2013).

\section{Referencias bibliográficas}

1. Levey A, Atkins R, Coresh J, Cohen E, Collins A, Eckardt K, et al. Enfermedad renal crónica como problema global en salud pública: Abordajes e iniciativas. Propuesta de la Kidney Disease Improving
Global Outcomes. Revista Kidney International. 2007; 3: 232-45.

2. Flores J, Alvo M, Borja H, Morales J, Vega J, Zúñiga $\mathrm{C}$, et al. Enfermedad renal crónica: Clasificación, identificación, manejo y complicaciones. Revista Médica de Chile. 2009; 13 (1): 137-177.

3. National-Kidney-Foundation. KDOQI Clinical practice guidelines for chronic kidney disease: evaluation, classification, and stratification. The National Kidney Foundation Kidney Disease Outcomes Quality Initiative; 2002.

4. National-Kidney-Foundation. KDOQI Clinical practice guideline for hemodialysis adequacy: 2015 update. The National Kidney Foundation Kidney Disease Outcomes Quality Initiative; 2015.

5. Colombia, Ministerio de la Protección Social, Programa de Apoyo a la Reforma de Salud. Guía para el manejo de la enfermedad renal crónica y modelo de prevención y control de la enfermedad renal crónica. Componente de un modelo de salud renal. Bogotá: Ministerio de la Protección Social; 2007.

6. Gotsadze G, Zoidze A, Rukhadze N. Household catastrophic health expenditure: evidence from Georgia and its policy implications. BMC Health Services Research. 2009: 9 (1): 1-9.

7. Toro W. Marco conceptual: enfermedades ruinosas o catastróficas (ERC) en el régimen contributivo del sistema de salud colombiano Capítulo 1. En: Modelo de simulación prospectiva de la demanda de servicios de salud para enfermedades de alto costo: Aplicación para una entidad promotora de salud colombiana [Internet]. Disponible en http://www. eumed.net/tesis-doctorales/wrtj/03.pdf.

8. Trevino-Becerra A. Insuficiencia renal crónica: enfermedad emergente, catastrófica y por ello prioritaria (editorial). Cirugía y Cirujanos. 2004; 72 (1): 3-4.

9. Aparicio C, Fernández A, Garrido G, Izquierdo E, Luque A. Calidad de vida percibida por niños con enfermedad renal crónica y por sus padres. Revista Nefrología. 2010; 30 (1): 103-9.

10. Yaffe K, Ackerson L, Tamura MK, Le Blanc P, Kusek JW, Sehgal AR, et al. Chronic kidney disease and cognitive function in older adults: Findings from the chronic renal insufficiency cohort cognitive study. Journal of the American Geriatrics Society. 2010; 58 (2): 338-45.

11. Varela D, Guarín M, Hincapié N, Hincapié S, Rodríguez K. Factores no tradicionales influyentes en la calidad de vida de los pacientes de hemodiálisis. Rev Colombiana Nefrología. 2014; 1 (1): 17-24.

12. Soriano S. Definición y clasificación de los estadios de la enfermedad renal crónica. Prevalencia. Claves para el diagnóstico precoz. Factores de riesgo de enfermedad renal crónica. Revista Nefrología. 2004; 24 (6): 27-34.

13. Alcázar R, Egocheaga, M, Orte L, Lobos J, González E, Álvarez F, Górriz J, Navarro J, Martín de Francisco A. Documento de consenso SEN-SemFYC sobre la enfermedad renal crónica. Revista de Nefrología. 2008; 28 (3): 273-82.

14. García P, Munévar M, Benavides C, Contreras K Primer trasplante renal en Colombia en paciente 
con infección por VIH. Acta Médica Colombiana. 2015; 40: 162-5.

15. Piedrahíta VM, Prada MC, Vanegas JJ, Vélez C, Serna LM, Serrano AK, et al. Causas de enfermedad renal crónica en niños atendidos en el Servicio de Nefrología Pediátrica del Hospital Universitario San Vicente de Paúl, de Medellín, Colombia, entre 1960 y 2010. Iatreia. 2011; 24 (4): 347-52.

16. Buitrago F, Calvo-Hueros JI, Gómez-Jiménez C, Canon-Barroso L, Suárez-González F, Robles NR. Hidden chronic renal insufficiency and cardiovascular events in patients with hypertension in a primary care center. Renal Failure. 2010; 32 (7): 757-65.

17. Shlipak MG, Fried LF, Stehman-Breen C, Siscovick D, Newman AB. Chronic renal insufficiency and cardiovascular events in the elderly: findings from the cardiovascular health study. The American Journal of Geriatric Cardiology. 2004; 13 (2): 81-90.

18. Wyburn KR, Horvath JS. Chronic renal insufficiency and renoprotective strategies. Internal Medicine Journal. 2003; 33 (5-6): 237-41.

19. Martínez F, Ordóñez I, García D. Deficiencias en el tratamiento de pacientes diabéticos que terminaron en enfermedad renal crónica. Acta Médica Colombiana. 2007; 32 (2).

20. Colombia, Ministerio de Salud y Protección Social, Ministerio de Hacienda y Crédito Público, Cuenta de Alto Costo: Fondo Colombiano de Enfermedades de alto Costo. Situación de la enfermedad renal crónica en Colombia, 2013 [Internet]. Bogotá: Ministerio de Salud y Protección Social; 2014 [acceso: 19 de abril del 2016]. Disponible en: https://www. minsalud.gov.co/sites/rid/Lists/BibliotecaDigital/ RIDE/INEC/CAC/SITUACION_DE_LA_ENFERMEDAD_RENAL_CRONICA_2013.pdf.

21. Santacruz P, Dorta L. La carga de la enfermedad renal crónica (ERC) sobre la mortalidad de la población. ¿Los datos disponibles reflejan la realidad? Nefrología. 2006; 26 (4), 419-420.

22. Rodríguez KA. Situación de la nefrología en Colombia. Asociación Colombiana de Nefrología e Hipertensión Arterial. 2009; 1(4): 5-17.

23. Roselli D, De Antonio R, Calderón C. Análisis económico de diálisis peritoneal comparada con hemodiálisis en pacientes con enfermedad renal crónica, diabética o hipertensiva. MedUnab. 2008; 11 (3): 201-5.

24. Colombia, Ministerio de Salud y Protección Social, Ministerio de Hacienda y Crédito Público, Cuenta de Alto Costo: Fondo Colombiano de Enfermedades de alto Costo. Situación de la enfermedad renal crónica en Colombia, 2012 [Internet]. Bogotá: Ministerio de Salud y Protección Social; 2014 [acceso: 19 de abril del 2016]. Disponible en: https:// cuentadealtocosto.org/site/images/Publicaciones/ Situacion $\% 20 \mathrm{de} \% 201 \mathrm{a} \% 20$ Enfermedad $\% 20$ Renal\%20Cronica\%20en\%20Colombia\%202012.pdf.

25. Colombia, Ministerio de Salud y Protección Social, Ministerio de Hacienda y Crédito Público. Cuenta de Alto Costo: Fondo Colombiano de Enfermedades de alto Costo. Situación de la enfermedad renal, hipertensión arterial y diabetes mellitus en Colombia, 2015 [Internet]. Bogotá:
Ministerio de Salud y Protección Social; 2015 [acceso: 19 de abril del 2016]. Disponible en: https://cuentadealtocosto.org/site/images/Situaci $\%$ C3\%B3n_de_la_Enfermedad_Renal_ Cr\%C3\%B3nica_en_Colombia_2015.pdf.

26. Hsu CY, Vittinghoff E, Lin F, Shlipak MG. The incidence of end-stage renal disease is increasing faster than the prevalence of chronic renal insufficiency. Annals of Internal Medicine. 2004; 141 (2): 95-101.

27. Colombiana de Salud. Guía de identificación de pacientes con enfermedad renal crónica: $2015-$ 2020 [Internet]. Bogotá: Colombiana de Salud; 2015 [acceso: 19 de abril del 2016]. Disponible en: http://www.colombianadesalud.org.co/MEDICINA/ Guia \%20estadio\%20falla\%20renal.pdf.

28. Ministerio de la Protección Social F. Modelo de prevención y control de la enfermedad renal crónica: Componente de un modelo de salud renal [Internet]. Bogotá: Ministerio de la Protección Social; 2005 [acceso: 19 de abril del 2016]. Disponible en: http://www.nuevalegislacion.com/files/susc/cdj/ conc/mod_pyc_ecr_r_3442_06.pdf.

29. Colombia, Ministerio de Salud y Protección Social, Ministerio de Hacienda y Crédito Público, Cuenta de Alto Costo: Fondo Colombiano de Enfermedades de Alto Costo. Informe de pacientes en terapia dialítica [Internet]. Bogotá: Ministerio de Salud y Protección Social; 2010 [acceso: 19 de abril del 2016]. Disponible en: https://cuentadealtocosto. org/site/images/TERAPIA_DIALITICA_EN_COLOMBIA_2010.pdf.

30. Lincoln C, Berlinguer G. Equidad en la salud en un mundo que marcha hacia la globalización. En: Evans T, Whitehead M, Diderichsen F, Bhuiya A, Wirth M, editores. Desafío a la falta de equidad en salud: de la ética a la acción. Washington: Fundación Rockefeller, Organización Panamericana de la Salud, Oficina Sanitaria Panamericana, Oficina Regional de la Organización Mundial de la Salud; 2002. p. 37-44.

31. Organización Mundial de la Salud. Dieta, nutrición y prevención de enfermedades crónicas [informe técnico]. [Internet]. Ginebra: 2003 [acceso: 19 de abril del 2016]. Disponible en: http://www.who.int/nutrition/publications/obesity/WHO_TRS_916_spa.pdf.

32. Organización Mundial de la Salud. Informe sobre la situación mundial de las enfermedades no transmisibles 2010. Resumen de orientación [Internet]. Ginebra: oms; 2011 [acceso: 19 de abril del 2016]. Disponible en: http://www.who.int/nmh/publications/ncd_report_summary_es.pdf.

33. Acosta P, Chaparro L, Rey C. Calidad de vida y estrategias de afrontamiento en pacientes con insuficiencia renal crónica sometidos a hemodiálisis, diálisis peritoneal o trasplante renal. Revista Colombiana de Psicología. 2008; 17 (1): 9-26.

34. Ibáñez-Beroiz B, Librero-López J, Peiró-Moreno S, Bernal-Delgado E. Shared component modelling as an alternative to assess geographical variations in medical practice: gender inequalities in hospital admissions for chronic diseases. BMC Medical Research Methodology. 2011; 11(1). 
35. Franco-Marina F, Tirado-Gómez L, VenadoEstrada A, Moreno-López J, Pacheco-Domínguez R, Durán-Arenas L, López- Cervantes M. Una estimación indirecta de las desigualdades actuales y futuras en la frecuencia de la enfermedad renal crónica terminal en México. Salud Pública de México [revista en internet]. 2011 [acceso: 19 de abril del 2016]; 53 (4). Disponible en: http://www. scielo.org.mx/scielo.php?script $=$ sci_arttext\&pid =S0036-36342011001000015.

36. Sen A. ¿Por qué la equidad en salud? Rev Panam Salud Pública. 2002; 11 (5-6).

37. Linares N, López O. La equidad en salud: Propuestas conceptuales aspectos críticos y perspectivas desde el campo de la salud colectiva. Medicina Social. 2008; 3 (3): 247-59.

38. Evans T, Whitehead M, Diderichsen F, Bhuiya A, Wirth M. Desafío a la falta de equidad en la salud: de la ética a la acción. Rev Inst Med Trop S Paulo [revista en internet]. 2003 [acceso: 19 de abril del 2016]; 45 (3). Disponible en: http://www.scielo.br/scielo.php? pid =S003646652003000300016\&script $=$ sci_arttext.

39. Ricardo AC, Yang W, Lora CM, Gordon EJ, Diamantidis CJ, Ford V, et al. Limited health literacy is associated with low glomerular filtration in the chronic renal insufficiency cohort (CRIC) study. Clinical Nephrology. 2014; 81 (1): 30-7.

40. Colombia, Congreso de la República de Colombia. Ley 100 por la cual se crea el sistema de seguridad social integral y se dictan otras disposiciones [Internet]. Bogotá: el Congreso; 1993. Disponible en: http://www.alcaldiabogota.gov.co/sisjur/normas/ Norma1.jsp? $\mathrm{i}=5248$.

41. Colombia, Ministerio de Salud. Resolución 5261 por la cual se establece el Manual de Actividades, Intervenciones y Procedimientos del Plan Obligatorio de Salud en el Sistema General de Seguridad Social en Salud. Bogotá: el Ministerio; 1994.

42. Colombia, Corte Constitucional. Sentencia T-760. Invoca la protección del derecho a la salud, concretamente, el acceso a servicios de salud que se requieren. Bogotá: Corte Constitucional; 2008 [Internet]. Disponible en: https://www.ramajudicial. gov.co/web/seguimiento-tutela-t_760-del-2008/ sentencia-t-760/08.

43. Colombia, Ministerio de Protección Social. Ley 1438 del 2011 por medio de la cual se reforma el Sistema General de Seguridad Social en Salud y se dictan otras disposiciones [Internet]. Bogotá: Ministerio de la Protección Social; 2011. Disponible en: http:// www.secretariasenado.gov.co/senado/basedoc/ ley_1438_2011.html.

44. Belalcázār I. La equidad en el Plan Obligatorio de Salud Colombiano: Una visión comparada [Internet]. Bogotá: Universidad Nacional de Colombia, Facultad de Derecho, Ciencias Políticas y Sociales; 2012 [acceso: 19 de abril del 2016]. Disponible en: http://www.bdigital.unal.edu.co/9019/1/isabeleugeniabelalcazarpe \%C3\%B1a.2012.pdf.

45. Ministerio de la Protección Social - Programa de Apoyo a la Reforma de Salud, Universidad Nacional de Colombia, Instituto de Investigacio- nes Públicas. Guías de promoción de la salud y prevención de enfermedades en la salud pública (tomo 2) [Internet]. Bogotá: el Ministerio; 2007 [acceso: 19 de abril del 2016]. Disponible en: https://www.minsalud.gov.co/Documentos $\% 20$ y $\% 20$ Publicaciones/GUIAS $\% 20$ DE $\% 20$ ATENCION $\% 20$ -томо \%20Dos.pdf.

46. Colombia, Ministerio de la Protección Social. Resolución 412 por la cual se establecen las actividades, procedimientos e intervenciones de demanda inducida y obligatorio cumplimiento y se adoptan las normas técnicas y guías de atención para el desarrollo de las acciones de protección específica y detección temprana y la atención de enfermedades de interés en salud pública. Bogotá: Ministerio de la Protección Social; 2000.

47. Colombia, Ministerio de la Protección Social. Resolución 2565 de 2007 por la cual se definen las enfermedades de alto costo. Bogotá: Ministerio de la Protección Social; 2007.

48. Colombia, Ministerio de Salud. Resolución 5261 por la cual se establece el Manual de Actividades, Intervenciones y Procedimientos del Plan Obligatorio de Salud en el Sistema General de Seguridad Social en Salud [Internet]. Bogotá: El Ministerio; 1994. Disponible en: https://www.minsalud.gov.co/ Normatividad_Nuevo/RESOLUCI\%C3\%93N\%20 5261\%20DE\%201994.pdf.

49. Colombia, Ministerio de la Protección Social. Resolución 3442 por la cual se adoptan guías de práctica clínica basadas en evidencia para la prevención, diagnóstico y tratamiento de los pacientes con VIH/ sida y enfermedad renal crónica y las recomendaciones de los modelos de gestión programática en VIH/sida y de prevención y control de la enfermedad renal crónica [Internet]. Bogotá: Diario Oficial, 2006. Disponible en: https://www.minsalud.gov. co/Normatividad_Nuevo/RESOLUCI \%C3\%93N\%20 3442\%20DE\%202006.pdf.

50. Colombia, Ministerio de la Protección Social. Acuerdo 245 para establecer una política integral para el manejo del alto costo, garantizar la adecuada atención de los pacientes afiliados a los regímenes contributivo y subsidiado, realizar vigilancia epidemiológica y mejorar la utilización de los recursos financieros del Sistema General de Seguridad Social. Salud Consejo Nacional de Seguridad Social en Salud. Bogotá: Diario Oficial; 2003.

51. Colombia, Ministerio de la Protección Social, Comisión de Regulación en Salud. Acuerdo 29 por el cual se sustituye el Acuerdo 028 del 2011 que define, aclara y actualiza integralmente el Plan Obligatorio de Salud [Internet]. Bogotá: Diario Oficial; 2011. Disponible en: https://www.minsalud.gov.co/salud/ POS/mi-plan/Normatividad $\% 20 y \% 20$ estudios $\% 20$ de $\% 20 \mathrm{mi} \% 20$ plan/Acuerdo $\% 20029 \% 20$ pos $\% 20$ 2012.pdf.

52. Colombia, Ministerio de la Protección Social. Resolución 1841. Plan Decenal de Salud Pública. Bogotá: el Ministerio; 2012.

53. Colombia. Ley 972 por la cual se adoptan normas para mejorar la atención por parte del Estado colombiano de la población que padece de enfer- 
medades ruinosas o catastróficas, especialmente el VIH/Sida. Bogotá: Diario Oficial; 2005.

54. Tonelli M, Gill J, Pandeya S, Bohm C, Levin A, Kiberd BA. Slowing the progression of chronic renal insufficiency. Canadian Medical Association Journal. 2002; 166 (7): 906-7.

55. Colombia, Ministerio de Salud y Protección Social, Ministerio de Hacienda y Crédito Público, Cuenta de Alto Costo: Fondo Colombiano de Enfermedades de alto Costo. Recomendaciones basadas en evidencia para la definición de: 1 . Criterios de inclusión a diálisis para pacientes con ERC estadio 5 y 2. Recomendaciones de inclusión a cuidado paliativo para pacientes con ERC estadio 5 sin indicación de terapia dialítica. Bogotá: Ministerio de Salud y Protección Social; 2013.

56. Colombia, Ministerio de la Protección Social. Resolución 1841 por la cual se adopta el Plan Decenal de Salud Pública 2012-2021. Bogotá: Diario Oficial 48811; 2013.

57. David I, Medina A, Martínez E. Enfermedades de alto costo en afiliados a un sistema institucional de aseguramiento y prestación de servicios de salud. Revista Facultad Nacional de Salud Pública. 2006; 24 (2): 98-104.

58. Chicaiza L. El mercado de la salud en Colombia y la problemática del alto costo. Revista Latinoamericana de Economía. 2002; 33: 164-87.

59. Mejía A, Sánchez A, Tamayo J. Equidad en el acceso a servicios de salud en Antioquia, Colombia. Revista de Salud Pública [revista en internet]. 2007 [acceso: 19 de abril del 2016]; 9 (1): 26-38. Disponible en: http://www.scielosp.org/pdf/rsap/v9n1/v9n1a04.pdf.

60. Colombia, Consejo Nacional de Seguridad Social en Salud. Acuerdo 260, 2004 [Internet]. Disponible en: http://www.saludcolombia.com/actual/htmlnormas/Acuer260.htm.

61. Colombia, Ministerio de Salud, Consejo Nacional de Seguridad Social en Salud. Acuerdo 30 por el cual se define el régimen de pagos compartidos y cuotas moderadoras dentro del Sistema General de Seguridad Social en Salud [Internet]. Bogotá, 1996. Disponible en: https://www.minsalud.gov. co/Normatividad_Nuevo/ACUERDO \%2030\%20 DE\%201996.pdf.

62. Colombia, Congreso de la República. Ley 1122 por la cual se hacen algunas modificaciones en el Sistema General de Seguridad Social en Salud y se dictan otras disposiciones [Internet]. Bogotá: Congreso de la República; 2007. Disponible en: http://www.secretariasenado.gov.co/senado/basedoc/ley_1122_2007.html.

63. Chicaiza L. Fallas del mercado de la Salud en Colombia: el caso de la insuficiencia renal crónica. Revista de Economía Institucional. 2005; 7 (12): 191-208.

64. Colombia, Ministerio de Salud, Consejo Nacional de Seguridad Social en Salud. Acuerdo 217 por el cual se establece un mecanismo para el reconocimiento de la desviación del perfil epidemiológico de la atención en salud del régimen contributivo [Internet]. Bogotá, 2001. Disponible en: http:// www.col.ops-oms.org/medicamentos/Documentos/ acu217_01.htm.
65. Nieto-Enciso LH. Análisis del comportamiento de la siniestralidad por enfermedades catastróficas en una empresa promotora de salud, Colombia. Rev Salud Pública. 2005; 7 (3): 293-304.

66. Colombia, Ministerio de Salud y Protección Social, Ministerio de Hacienda y Crédito Público, Cuenta de Alto Costo: Fondo Colombiano de Enfermedades de alto Costo. El pago por resultados como una estrategia que aborda los acuerdos de riesgo compartido en Colombia: iUna opción posible! Boletín de Información Técnica Actualizada [publicación en internet]. 10 de junio del 2015 [acceso: 19 de abril del 2016]; edición especial: 1-6. Disponible en: https://cuentadealtocosto.org/ site/images/Bolet \%C3\%ADn \%20especial \%20 Riesgo\%20compartido.pdf.

67. Sanabria-Arenas M, Paz-Wilches J, Laganis-Valcarcel S, Muñoz-Porras F, López-Jaramillo P, VesgaGualdrón J, et al. Inicio de diálisis y mortalidad en una población con enfermedad renal crónica en Colombia. Rev Fac Med. 2015; 63 (2): 209-16.

68. Dehesa E. Enfermedad renal crónica: definición y clasificación. Revista Medigraphic. 2008; 3 (3): 73-8.

69. Martínez M, Plazas M, Barajas G, Bravo A, González C, Rodríguez A, et al. Factores de riesgo para enfermedad renal crónica en pacientes que asisten a consulta de medicina interna. Acta Médica Colombiana. 2013; 38 (4): 228-32.

70. Colombia, Ministerio de Salud y Protección Social, Ministerio de Hacienda y Crédito Público, Cuenta de Alto Costo: Fondo Colombiano de Enfermedades de alto Costo. Indicadores para seguimiento al trasplante renal en Colombia. Bogotá: Ministerio de Salud y Protección Social; 2012.

71. Colombia, Ministerio de Salud y Protección Social, Ministerio de Hacienda y Crédito Público, Cuenta de Alto Costo: Fondo Colombiano de Enfermedades de alto Costo. Indicadores mínimos para seguimiento de niños con enfermedad renal crónica en diálisis o trasplante renal en Colombia. Bogotá: Ministerio de Salud y Protección Social; 2010.

72. Colombia, Ministerio de Salud y Protección Social, Ministerio de Hacienda y Crédito Público, Cuenta de Alto Costo: Fondo Colombiano de Enfermedades de alto Costo. Indicadores mínimos para evaluar los resultados clínicos en pacientes incluidos en programas de nefroprotección para estadios 1-4 y 5 sin diálisis. Bogotá: Ministerio de Salud y Protección Social; 2013.

73. Sánchez P, Acuna L, Soler L, Torres L, Barrera A. Indicadores de nefroprotección: herramienta para evaluar resultados en gestión de riesgo para pacientes con enfermedad renal crónica, hipertensión y/o diabetes mellitus en Colombia. Value Health. 2015; 18 (7).

74. Colombia, Ministerio de Hacienda y Crédito Público, Ministerio de la Protección Social. Resolución 4700 por la cual se definen la periodicidad, la forma y el contenido de la información que deben reportar las entidades promotoras de salud y las demás entidades obligadas a compensar para la operación de la cuenta de alto costo. Bogotá: Diario Oficial $n^{\circ}$. 47190; 2008. 
75. Colombia, Ministerio de la Protección Social, Ministerio de Hacienda y Crédito Público. Resolución 3215 por la cual se fijan los mecanismos de cálculo para definir los montos de aporte por parte de las empresas promotoras de salud EPs del régimen contributivo y subsidiado y entidades obligadas a compensar, EOC, y de distribución de recursos de la cuenta de alto costo, en el caso de la terapia de reemplazo renal por enfermedad renal crónica, ERC. Bogotá: Diario Oficial n. 46768; 2007.

76. Colombia, Ministerio de Salud y Protección Social, Ministerio de Hacienda y Crédito Público, Cuenta de alto Costo: Fondo Colombiano de Enfermedades de alto Costo. Indicadores de diálisis por prestadores [Internet]. Bogotá: Ministerio de Salud y Protección Social; 2012 [acceso: 19 de abril del 2016]. Disponible en: https://cuentadealtocosto. org/site/images/Publicaciones/Indicadores_IPSERC_jun2012.pdf.

77. Saad Acosta C, Sepúlveda G, Ibáñez E, Flórez C, Herrán M, Márquez N, et al. Adherencia de los pacientes con insuficiencia renal crónica a las sesiones de hemodiálisis del Hospital Militar Central, Bogotá, D. C., 2006-2007. Revista Colombiana de Enfermería. 2008; 3 (3): 61-7.

78. Rodríguez M, Varela M, Rincón H, Velasco M, Caicedo D, Méndez F, et al. Prevalencia y factores asociados a la adherencia al tratamiento no farmacológico en pacientes con hipertensión y diabetes en servicios de baja complejidad. Revista Facultad Nacional de Salud Pública. 2015; 33 (2): 192-9.

79. Ortiz L, Cardona T, Berona Y. Caracterización de la demanda atendida por enfermedad renal crónica y sus precursoras de Antioquia, Colombia 2011. Medellín: Universidad de Antioquia; 2015.

80. Tamayo DC, Tono TM. Acceso y calidad de los servicios de salud para los pacientes con diabetes mellitus en Colombia: revisión sistemática de la literatura. Observatorio de Diabetes en Colombia [Internet] [acceso: 19 de abril del 2016]. Disponible en: http://www.odc.org.co/files/posters/ Posters_para_presentar.pdf.

81. Coronado CY, Lombo JC, Correa I, Quintero N. Características clínicas y demográficas de los pacientes incidentes en diálisis crónica y su relación con el ingreso programado a diálisis. Acta Médica Colombiana. 2013; 38 (3): 138-42.

82. López SM. Nivel de adherencia al tratamiento farmacológico en pacientes con enfermedad renal crónica en diálisis, en una IPs de la ciudad de Cali. Santiago de Cali: Universidad del Valle; 2012.

83. Carrillo AJ. Análisis de la capacidad de autocuidados en pacientes en diálisis peritoneal. Enferm Nefrol. 2015; 18 (1): 31-40.
84. Tessone A, Gottlieb S, Barbash IM, Garty M, Porath A, Tenenbaum A, et al. Underuse of standard care and outcome of patients with acute myocardial infarction and chronic renal insufficiency. Cardiology. 2007; 108 (3): 193-9.

85. Colombia, Ministerio de la Protección Social. Resolución 4918 por la cual se fijan los mecanismos e instrumentos de ejecución y los criterios de distribución de los recursos contemplados en la Ley Anual de Presupuesto para la vigencia 2009, destinados a financiar actividades del Plan Nacional de Salud Pública orientadas a promover las acciones de diagnóstico temprano y reducción de la nefropatía diabética e hipertensiva [Internet]. Bogotá: Ministerio de la Protección Social; 2009 [acceso: 19 de abril del 2016]. Disponible en: https:// cuentadealtocosto.org/site/images/Resolucion $\% 20$ 4918\%20-\%202009\%20-\%20ERC.pdf.

86. Colombia, Ministerio de la Protección Social. Decreto 2699 por el cual se establecen algunas normas relacionadas con el Sistema General de Seguridad Social en Salud y se dictan otras disposiciones. Bogotá: Diario oficial nº. 46.688; 2007.

87. Molina D. Propuesta en prevención del riesgo cardiovascular. Revista Colombiana de Cardiología. 2008; 15 (5): 1-5.

88. Sanabria M, Rodríguez K, Sánchez R, Astudillo K, Camargo D, Bunch A. Frecuencia y costos de hospitalización en una población de pacientes en diálisis en Colombia. Rev Fac Med. 2012; 60 (4): 293-301.

89. Jeannette A, Ruiz F. Determining factors of catastrophic health spending in Bogotá, Colombia. Int J Health Care Finance Econ. 2011; 11 (2): 83-100.

90. Organización Internacional del Trabajo (OIT), Organización Panamericana de la Salud (ops). El gasto de bolsillo en salud en América Latina y el Caribe: Razones de deficiencia para la extensión de la protección social en salud [Internet]. México: оIт, ops; 1999 [acceso: 19 de abril del 2016]. Disponible en: https://www.seguroscaracas.com/ paginas/biblioteca_digital/PDF/ucv/regimenes-ss/ elgastodebolsilloensaludenamericalatinayelcaribe. pdf 1999. 1-29.

91. Colombia, Ministerio de Salud y Protección Social. Ley 1751 por medio de la cual se regula el derecho fundamental a la salud y se dictan otras disposiciones. Bogotá: el Ministerio; 2015.

92. Colombia, Ministerio de Salud y Protección Social. Resolución 429 por medio de la cual se adopta la Política de Atención Integral en Salud. Bogotá: el Ministerio; 2016. 